\title{
FERMI LEVEL POSITION AT SEMICONDUCTOR SURFACES ${ }^{*}$
}

\author{
C. A. Mead \\ California Institute of Technology, Pasadena, California \\ and \\ W. G. Spitzer \\ Bell and Howell Research Center, Pasadena, California \\ (Received 2 May 1963)
}

There have been several recent reports of barrier height studies on metal-semiconductor interfaces. ${ }^{1-7}$ Metals of widely different work functions evaporated onto $\mathrm{Si}$ and GaAs surfaces indicated that in each case the energy difference between the semiconductor conduction band edge and Fermi level at the interface, $\varphi_{B n}$, was essentially independent of the metal, which indicates that the Fermi level is fixed by surface states. In the present work barrier height measurements have been made on a number of zincblende semiconductors to determine (a) if the barriers are in all cases determined by surface states, and (b) the relation between the Fermi energy at the interface and the band gap $E_{g}$.

The present results along with those previously reported are summarized in Table I. In all cases, the barrier heights were essentially independent of the metal. ${ }^{8}$ In no case was the relation $E_{g}=\varphi_{B n}+\varphi_{B p}$ violated, where $\varphi_{B p}$ is the Fermi energy with respect to the valence band edge. For those materials where both $300^{\circ} \mathrm{K}$ and $77^{\circ} \mathrm{K}$ measurements could be made, $\varphi_{B p}$ was independent of temperature, and the change

Table I. Barrier heights for Au on several semiconductors. Si taken from reference 1 and GaP from reference 3. Values are for room temperature unless otherwise specified. Where $\varphi_{B P}$ was not available directly, $E_{g}-\varphi_{B n}$ was used.

\begin{tabular}{|c|c|c|c|c|c|}
\hline $\begin{array}{l}\text { Periodic } \\
\text { table row }\end{array}$ & Semiconductor & $E_{g}$ & ${ }^{\varphi} B n$ & $\varphi_{B p}$ & $\varphi_{B p / E g}$ \\
\hline 2 & $\mathrm{Si}$ & 1.12 & 0.78 & $\cdots$ & 0.3 \\
\hline 3 & $\mathrm{Ge}$ & 0.66 & 0.46 & $\cdots$ & 0.3 \\
\hline $3-3$ & GaAs & 1.35 & 0.93 & 0.46 & 0.35 \\
\hline $4-4$ & $\operatorname{InSb}\left(77^{\circ} \mathrm{K}\right)$ & 0.24 & 0.17 & $<0.1$ & 0.3 \\
\hline $3-2$ & GaP & 2.24 & $\cdots$ & 0.72 & 0.3 \\
\hline $2-4$ & $\mathrm{AlSb}$ & 1.6 & $\cdots$ & 0.56 & 0.35 \\
\hline $4-2$ & InP & 1.26 & 0.5 & 0.76 & 0.6 \\
\hline $3-4$ & $\mathrm{GaSb}$ & 0.70 & 0.6 & $<0.1$ & 0.15 \\
\hline $4-3$ & InAs $\left(77^{\circ} \mathrm{K}\right)$ & 0.43 & $<0.1$ & 0.48 & 1.1 \\
\hline
\end{tabular}

in $\varphi_{B n}$ was equal to the change in $E_{g}$.

The values of $\varphi_{B n}$ and $\varphi_{B p}$ were determined primarily by the voltage variation of the capacitance $e^{1,9}$ and checked, where possible, by the forward diode characteristic and the spectral dependence of the photoresponse. ${ }^{2}$ Surfaces were prepared by vacuum cleavage or by chemical polishing ${ }^{10}$ followed by an $\mathrm{HF}$ rinse, and in those cases where good samples were obtained by both techniques the results agreed. For small-E $E_{g}$ materials, notably InSb and InAs, barriers on cleaved surfaces exhibited excessive reverse leakage and could not be used.

A most remarkable feature of the results is that for most of the materials the Fermi level is fixed the same fraction of the band gap above the valence band edge. This relation holds for an order of magnitude variation in $E_{g}$. Compounds which are exceptions are those containing elements from different rows of the periodic

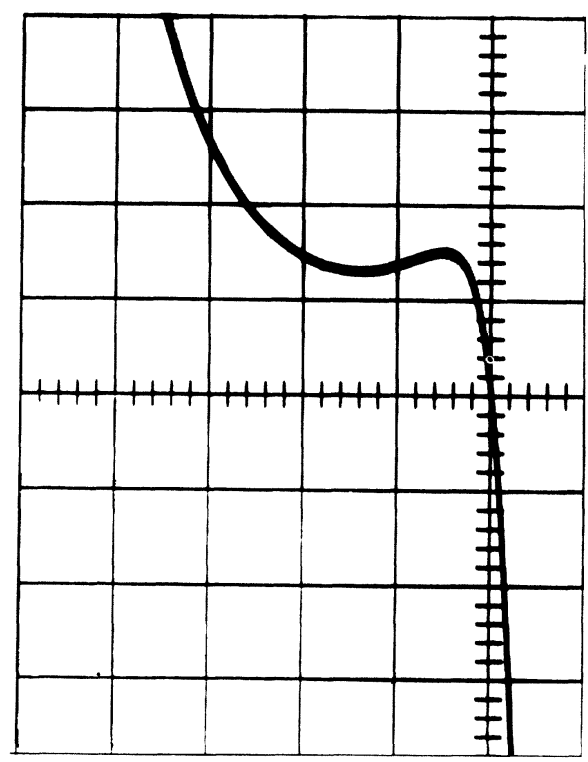

FIG. 1. $I-V$ characteristic for 0.01-in. diameter Au on degenerate $p$-type InAs at $77^{\circ} \mathrm{K}$. Vertical scale 0.2 $\mathrm{mA} / \mathrm{cm}$. Horizontal scale $-0.05 \mathrm{~V} / \mathrm{cm}$. The squares are $1 \mathrm{~cm}^{2}$. 
table and in which there is at least one heavy element. Perhaps the most surprising value of $\varphi_{B p}$ is that for InAs where there is not only an inversion layer on the $p$-type material but the Fermi level at the interface is actually above the conduction band edge. A striking confirmation of this result is seen in the $I-V$ characteristic (Fig. 1) of a unit prepared on degenerate $p$-type InAs. The negative resistance region can only occur if the Fermi level is in the conduction band at the surface. Energy values determined from peak and valley voltages agree with those obtained from capacitance data.

The authors wish to express their appreciation to H. M. Simpson, R. K. Willardson, and W. P. Allred for their aid in the above work.
*Work supported in part by the U. S. Office of Naval Research and the International Telephone and Telegraph Company.

${ }^{1}$ R. J. Archer and M. M. Atalla, Ann. N.Y. Acad. Sci. 101, 697 (1963).

${ }^{2}$ C. R. Crowell, W. G. Spitzer, L. E. Howarth, and E. E. LaBate, Phys. Rev. 1272006 (1962).

${ }^{3}$ C. R. Crowell, W. G. Spitzer, and H. G. White, Appl. Phys. Letters 1,3 (1962).

${ }^{4}$ G. W. Mahlman, Phys. Rev. Letters 7408 (1961).

${ }^{5}$ R. Williams, Phys. Rev. Letters $\underline{8}, 402$ (1962).

${ }^{6}$ C. A. Mead and W. G. Spitzer, Appl. Phys. Letters 2, 74 (1963).

${ }^{7}$ W. G. Spitzer and C. A. Mead (to be published).

${ }^{8}$ For GaP. R. A. Logan (private communication).

${ }^{9}$ M. A. Goodman, J. Appl. Phys. 34, 329 (1963).

${ }^{10} \mathrm{C}$. S. Fuller and H. W. Allison, J. Electrochem. Soc. 109,880 (1962).

\title{
EVIDENCE FOR THE ROLE OF DONOR STATES IN GaAs ELECTROLUMINESCENCE
}

\author{
F. L. Galeener \\ National Magnet Laboratory,* Massachusetts Institute of Technology, Cambridge, Massachusetts \\ and
}

G. B. Wright, W. E. Krag, T. M. Quist, and H. J. Zeiger

Lincoln Laboratory, ${ }^{\dagger}$ Massachusetts Institute of Technology, Lexington, Massachusetts

(Received 25 March 1963)

The mechanism of the transition responsible for maser action in GaAs diodes has been the subject of lively interest since the first report of very efficient radiation from GaAs diodes. ${ }^{1}$ We wish to report some observations on the effect of a high magnetic field on this emission, which may give some clue to the nature of the transition involved. It will be shown that the observed shift of emission energy with magnetic field can be approximately described by assuming that the transition proceeds through the ground states of donors in GaAs.

The experiments were performed on maser diodes and incoherent diodes at liquid helium temperatures and on incoherent diodes at liquid nitrogen temperatures. These diodes were zincdiffused GaAs maser diodes ${ }^{2}$ whose net $n$-type impurity concentration was typically $(2-5) \times 10^{17}$ $\mathrm{cm}^{-3}$. They were oriented with the magnetic field in the plane of the junction. The incoherent diodes were pulsed at $13 \mathrm{cps}$ with a peak current of $80 \mathrm{~mA}$, while the maser diodes were pulsed at $300 \mathrm{cps}$ with a peak current of $2.75 \mathrm{~A}$. The field was supplied by a Bitter-type solenoid which gave $90 \mathrm{kG}$ at 10 kiloamperes. A typical recorder trace for a maser diode is shown in Fig. 1. As the field is increased, the emission energy shifts, new peaks appear, and others disappear. $^{3}$

The spacing of electromagnetic modes of the

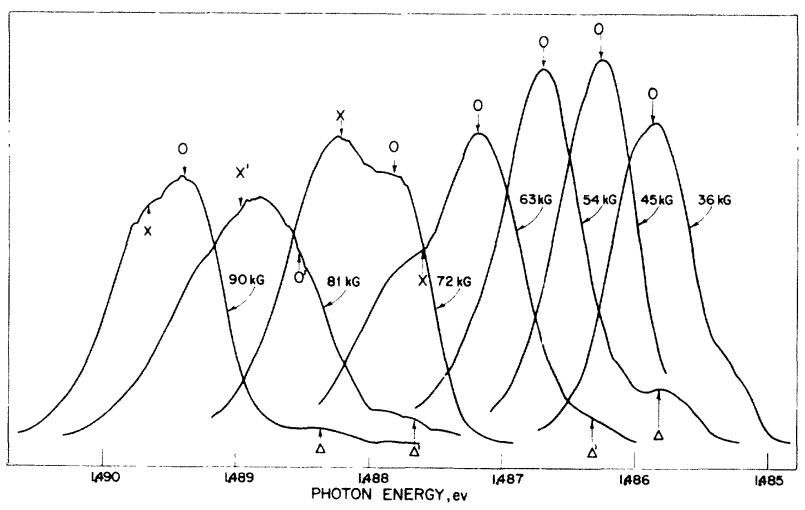

FIG. 1. Recorder trace of GaAs maser emission. Symbols identify the three different series observed. Primed symbols indicate the position of series peaks calculated from the quadratic field dependence. 